\title{
Effect of Population Doublings on Differentiation Capacity of Human Adipose-Derived Stem Cells: Establishing Standard Guidelines for Clinical Translational Applications
}

\author{
Research Article
}

Plastini MA, Kappy N, Chang S, Harris WM, Ortiz T, Zhang P, Carpenter JP, Brown SA*

Department of Surgery, Cooper University Hospital and Cooper Medical School of Rowan University, Camden, NJ, USA.

\section{Abstract}

\begin{abstract}
Human adipose-derived stem cells (ASCs) are providing promise in clinical soft tissue replacement and repair. This study investigated the effect of multiple population doublings (PD) on ASCs growth rates and cell specific differentiation capacities (DC) to evaluate potential inter-individual specimen growth rates and DC for in vitro cell expansion use in future clinical applications. A decreased cell growth rate and DC were observed in long term cell culture when PD reached to 10.1. Early PD specimens with PD range from 1.6 to 6.2 showed the average population doubling time (PDT) as 5.1 days. There was no significant difference in ASCs growth rates among individual specimens $(\mathrm{n}=4, \mathrm{p}=0.184)$. The DC over PD range 1.6 to 6.2 also showed no significant changes (osteogenic, $n=3, p=0.472$; chondrogenic, $n=3, p=0.878$; adipogenic, $n=3, p=0.256$ ). Our preliminary data showed the importance of PD for in vitro ASCs expansion. The cell growth rate and DC of ASCs were relatively stable up to PD 6.2. Beyond this PD, there was a trend of decreased growth rate and DC. Therefore, our study suggested that future in vitro expansion of ASCs for clinical application should consider limiting expansion of ASCs to PD 6.2 or less to ensure the cell quality. Use of specific PD instead of the non-specific passage numbers would be a better standardization of in vitro ASCs expansion for their clinical translational applications.
\end{abstract}

Keywords: Adipose-Derived Stem Cells; Population Doubling; Differentiation; Cell Growth.

\section{Background}

Many organs and tissue systems of the body contain a portion of undifferentiated, multipotent progenitor cells. These cells can be stimulated to differentiate into specific lineages of cells in the face of physical or biological stimuli $[1,2]$. These cells are located indifferent types of tissues including bone marrow, skeletal muscle, and adipose tissue [1, 3, 4]. Recent research has focused on these cells ability to differentiate not only into their native tissue, but along other lineages as well [5-7].

Fat grafting has long been utilized to fill in soft tissue defects both adjacent to the site of harvest and at distant sites [8]. Adipose derived stem cells (ASCs) are easily acquired through liposuction techniques, and possess the ability to differentiate along multiple cell lineages [5, 9-11]. ASCs ease of acquisition has led to intense research into their regenerative potential at the site of large areas of tissue loss [4]. Despite the higher yield and easier methods of harvest compared to other stem cell populations derived from muscle and bone marrow [12], ASCs number following initial harvest is often insufficient for bench, animal, or clinical experiments, therefore in vitro expansion is often necessary [3, 13].

The amount of in vitro expansion that these cells are subjected to is extremely variable and usually reported as a non-specific passage number [3, 13-19]. Dependent on the plating density and the confluence at which cells are harvested, the amount of population doublings (PDs) that a population of ASCs undergoes before reaching confluence is extremely variable and are unknown from many publications. This leads to inconsistencies in the amount of time and thus number of PDs that a cell population is subjected to before utilization in in vitro or in vivo models.

Previous research has demonstrated that as in vitro expansion of

\footnotetext{
*Corresponding Author:

Spencer A. Brown, Ph. D

Department of Surgery, Cooper University Hospital and Cooper Medical School of Rowan University, Camden, NJ, USA.

Tel: $856-7579646$

Fax: 856-7579647

E-mail: Brown-spencer@cooperhealth.edu

Received: July 21, 2016

Accepted: August 24, 2016

Published: August 26, 2016

Citation: Plastini MA, Kappy N, Chang S, Harris WM, Brown SA (2016) Effect of Population Doublings on Differentiation Capacity of Human Adipose-Derived Stem Cells: Establishing Standard Guidelines for Clinical Translational Applications. Int J Stem Cell Res Transplant, S2:001, 1-7. doi: http://dx.doi.org/10.19070/2328-3548-SI02001

Copyright: Brown SA ${ }^{\circ}$ 2016. This is an open-access article distributed under the terms of the Creative Commons Attribution License, which permits unrestricted use, distribution and reproduction in any medium, provided the original author and source are credited.
} 
ASCs is carried out for increasing time periods the cell population will exhibit both genetic and phenotypic changes [14, 16]. With increased passaging and the subsequently increased PDs, ASCsgrowth kinetics and ability to differentiate along varying cell lineages, or differentiation capacity (DC), is also affected [3, 14, 20]. Bieback et al., demonstrated that adipogenic differentiation rates were decreased with increased PD levels of 12-43.4 as compared to the baseline PD 4-5.7 [20].

Despite the huge regenerative potential ASCs present, human clinical trials have been limited [21]. One of the biggest hurtle preventing the wide spread clinical application of ASCs is the need to keep ASCs minimally manipulated prior to clinical application. In the United States, the US U.S Food Drug Administration (FDA) has authority over human cells, tissue, and cellular products, and thus over ASCs clinical use [22]. According to FDA regulations, homologous use of a cell suspension has to be minimally manipulated with little or no changes from the native cell phenotype [FDA 21 CFR 1271] which details testing, screening, shipping, and storage procedures. If a cell or tissue product is manipulated beyond what is considered minimal, then it falls under the category of a biologic drug which requires phase I, II, and III clinical trials and be granted a biological license [22]. This process can cost millions of dollars and often requires years to complete. The ability to classify ASCs as a cell therapy under regulation 21 CFR 1271 which is only subject to adherence togood manufacturing guidelines greatly simplifies the process of clinical application. Given the possibility and potential benefit of maintaining ASCs as a 'minimally manipulated' cell population, standardization of the in vitro expansion that ASCs are subject to prior to future clinical translational applications would be required.

This study specifically examined the effect of PD on both PDT and DC with the goal of establishing guidelines under which future safe in vitro expansion can be undertaken prior to clinical application.

\section{Methods}

\section{Isolation and preparation of ASCs}

Following Cooper University Hospital Institutional Human Safety and Review Board approval and consent from individual patients, human adipose tissue was obtained from three subjects. Subjects were all female, aged 29 to 51 who underwent elective breast reduction or in the case of one patient, elective thigh liposuction as well as breast reduction at Cooper University Hospital between August and October of 2015. Adipose tissue was obtained either via tumescent liposuction or en-bloc resection from the breasts of two patients, and the breasts as well as the thigh of the third patient. Any tissue obtained via en-block resection was minced whereas tissue harvested using liposuction was processed in its liquid/slurry form. All samples were washed with $37^{\circ} \mathrm{C}$ phosphate buffered saline (PBS) and weighed. The samples were incubated in $0.1 \%$ type I Collagenase (Worthington, Lakewood, NJ) for one hour. Ten $\mathrm{ml}$ of M199 with 10\% fetal bovine serum (FBS) was added to inactivate the collagenase and the resulting cell suspension was centrifuged at $1500 \mathrm{RPM}, 4^{\circ} \mathrm{C}$ for 10 minutes. The resultant cell pellet was suspended in M199 (Cellgro, Manassas, VA) and centrifuged at $1200 \mathrm{RPM}, 4^{\circ} \mathrm{C}$ for 5 minutes. This pellet was briefly (10 seconds) suspended in $1 \mathrm{ml}$ sterile, double distilled water to rupture RBCs, followed by the addition of M199 cell culture medium and filtered through a $100 \mu \mathrm{m}$ filter and centrifuged at $800 \mathrm{RPM}, 4^{\circ} \mathrm{C}$ for 5 minutes to obtain the stromal vascular fraction (SVF). An initial cell count of the SVF was determined using hemocytometer. The SVF cells were then plated at $1 \times 10^{6}$ in M199 (Gibco, Grand Island, NY) and10\% FBS and incubated overnight at $37^{\circ} \mathrm{C}$ in $5 \% \mathrm{CO}_{2}$ with room air. The next day, nonadherent cells were washed away with PBS and the attached cells were collected using $0.25 \%$ trypsin-EDTA solution. These fresh isolated ASCs were transferred to new cell culture containers based on experimental design as described below.

\section{Cell counting and cell population doubling}

Freshly isolated ASCs were platedin triplicate at seeding densities of 500 cells $/ \mathrm{cm}^{2}, 1000$ cells $/ \mathrm{cm}^{2}, 2500$ cells $/ \mathrm{cm}^{2}$, and 5000 cells $/ \mathrm{cm}^{2}$. These plated were then monitored daily using light microscopy and sub-cultured at 90\% confluence. Total cell numbers were determined by hemocytometer. Cell population doubling (PD) was calculated using the following equation:

$$
\mathrm{PD}=\frac{\operatorname{Ln}\left(\frac{\text { Cell number obtained }}{\text { Cell number seeded }}\right)}{\operatorname{Ln} 2}
$$

Cell population doubling time (PDT) was calculated as below:

$$
\mathrm{PDT}=\frac{\left(t_{2}-t_{1}\right)}{\mathrm{PD}}
$$

Where $\mathrm{t} 1$ and $\mathrm{t} 2$ are the beginning and end of the exponential phase, respectively.

\section{ASCs differentiation and assessing differentiation potential}

A subgroup of ASCs at different PD levels was subjected to osteogenic, adipogenic, and chondrogenic differentiation media. ASCs at $80 \%-90 \%$ confluency were incubated in StemPro ${ }^{\circledR}$ osteogenesis, adipogenesis, and chondrogenesis differentiation medium (Invitrogen, Carlsbad, CA) for two to three weeks. Alizarin Red, Oil Red-O, and Alician blue dyes were used to assess osteogenic, adipogenic, and chondrogenic differentiation, respectively. Alizarin Red was added to fixed cells that had been cultured in osteogenic medium for at least 21 days. Oil Red-O and Alician blue dyes were added to cells that had been cultured for at least 14 days in adipogenic medium and chondrogenic medium, respectively. To extract the dye, different reagents where utilized to solubilize the target dye. Briefly, the Alizarin Red, Oil Red-O, and Alician blue dyes were extracted using 10\% acetic acid, 100\% isopropanol, and 6M guanidine hydrochloride, respectively. The optical density (OD) of the extracted dye quantified using the following wavelengths: $405 \mathrm{~nm}$ for Alizarin Red, $495 \mathrm{~nm}$ for Oil Red-O, and $595 \mathrm{~nm}$ for Alician blue. Fold changes between undifferentiated controls and differentiated samples were compared.

\section{Data analysis}

All experiments were performed with three repeats for each sample. The quantitative data were presented as mean \pm standard error. Student's $t$-test and ANOVA test were used to assess statisti- 
cal significance. A p-value of $\leq 0.05$ was considered significant for all experiments.

\section{Results}

Long term effect of PD on ASCs cell growth and stem cell differentiation capability

One cell line was continuously cultured up to 99 days to measure the long-term effects of PD on ASCs cell growth and stem cell differentiation capability (Figure 1). This cell line was examined at four different PD stages as PD2.2, PD5.0, PD8.0 and PD 10.1. The cell population doubling time (PDT) was used to evaluate cell growth rate. A high PDT indicated a slow cell growth rate. PDT at PD8.0 and PD10.1 were higher than PDT at PD2.2 and PD5.0 (panel A). The stem cell differentiation capability was measured for adipogenic, osteogenic, and chondrogenic differentiations at PD 5.0 and PD 10.1 respectively. The representative staining images showed a declining trend of staining at PD10.1 group than the PD5.0 group (panel B). Three individual cell cultures were performed at both PD groups for each differentiation and the intensity of specific staining was used to quantify corresponding differentiation (panel C). The ratio of Alizarin Red staining for osteogenic differentiation capability decreased from 5.6 to 1.8 (n $=3, \mathrm{p} \leq 0.001)$. The ratio of Alician blue staining for chondrogenic differentiation decreased from 2.4 to $1.3(\mathrm{n}=3, \mathrm{p}=0.001$ ), while the ratio of Oil red-O staining for adipogenic differentiation slightly changed from 1.6 to 1.4 ( $n=3, p=0.293$ ).
This pilot long-term study showed a negative effect of higher PD (like 10.1) on ASCs cell growth and differentiation capabilities. Although only one cell line was studied in this experiment, there were triplicates for each sample and two differentiation capabilities (osteogenic and chondrogenic) were decreased at PD10.1. The experimental plan was then to focus on the earlier PD ASCs populations to determine optimal expansion conditions in which osteogenic and chondrogenic changes were minimal compared to PD 0.

\section{The short term effect of PD on ASCs cell growth}

Four freshly isolated ASCs were cultured at seeding density as 500 cells $/ \mathrm{cm}^{2}, 1000$ cells $/ \mathrm{cm}^{2}, 2500$ cells $/ \mathrm{cm}^{2}$, and 5000 cells $/ \mathrm{cm}^{2}$ respectively. Daily observations demonstrated that lower density seeded cells exhibited more spindle-like characteristics compared to those seeded at higher cell densities (Figure 2, panels A and B). The lower seeding densities required longer culture times for the cells to reach confluency. The 5000 cells $/ \mathrm{cm}^{2}$ seeding density group required 12.3 days to become confluent with a corresponding cell PD of 1.6. The 2500 cells $/ \mathrm{cm}^{2}$ seeding density group required 18.5 days to reach confluency with a PD of 3.8. The PD for the 1000 cells $/ \mathrm{cm}^{2}$ and the 500 cells $/ \mathrm{cm}^{2}$ seeding density groups were 4.9 and 6.2 , respectively. The average PDT was 6.1 days for the 5000 cells $/ \mathrm{cm}^{2}$ seeding group, 5.1 days for the 2500 cells $/ \mathrm{cm}^{2}$ seeding group, 4.9 days for the 1000 cells $/ \mathrm{cm}^{2}$ seeding group and 6.2 days for the 500 cells $/ \mathrm{cm}^{2}$ seeding group. No significant differences in ASCs growth rates among individual specimens were observed overall for PD 1.6-6.2 $(n=4, p=0.184)$

Figure 1. The effect of high population doubling number on the cell growth and differentiation capability of Adipose-derived stem cells (ASCs). One ASCs cell line was continuously sub-cultured for up to population doubling (PD) at 10.1. The effects of PD on cell growth and differentiation capability were measured within the same cell line at different PD. Panel A: cell population doubling time at different PD number; Panel B: representative staining images for adipogenic, osteogenic, and chondrogenic differentiations at PD 5.0 and PD 10.1 respectively; Panel C: bar graph for quantified analysis of ASCs differentiation capabilities at PD 5.0 and PD 10.1. For each differentiation, three individual measurements were done. Significant decreases of differentiation capabilities occurred for osteogenic differentiation $(n=3, p \leq 0.001)$ and chondrogenic differentiation $(n=3, p=0.001)$, while the change of adipogenic differentiation was not significant $(n=3, p=0.293)$. $(*$ denotes values with statistical significance).

A

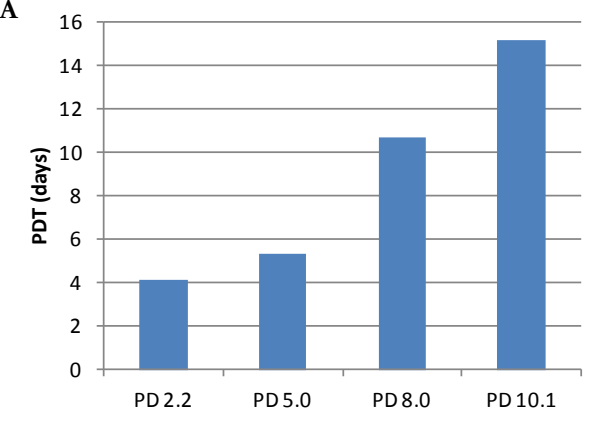

C

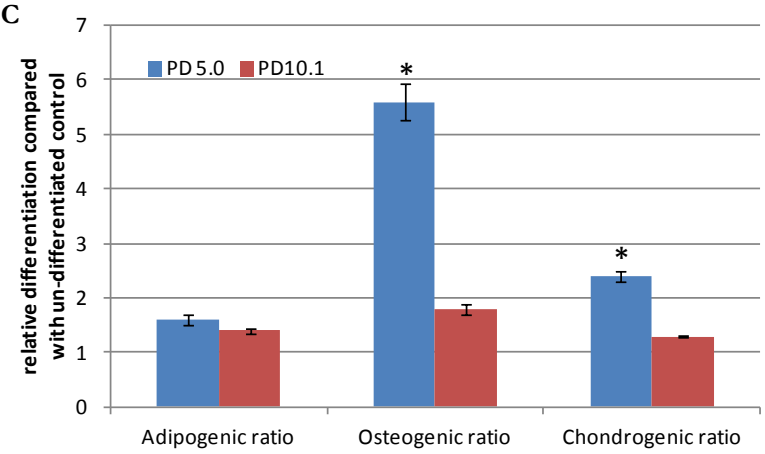

B

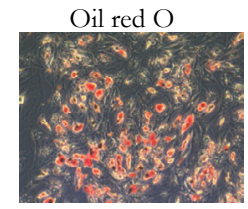

PD 10.1
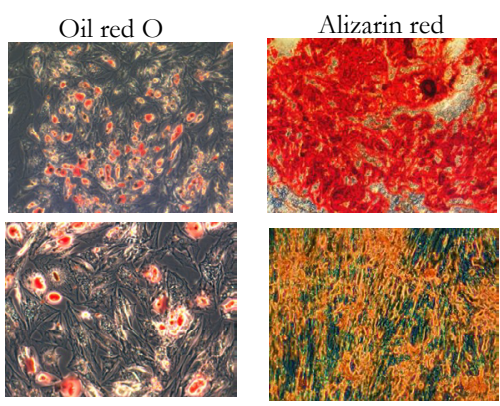

Alician blue
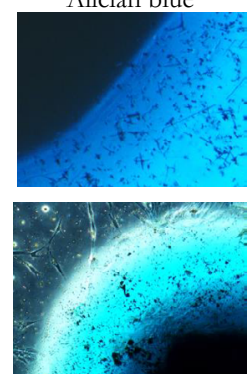
Figure 2. The cell growth of fresh isolated Adipose-derived stem cells (ASCs) at different seeding density.Panel A: representative cell images at day 3 post-isolation; Panel B: representative ASCscell images at day 6 post-isolation; Panel C: bar graph of data from four individual cell lines to show the population doubling time (PDT) which demonstrated no significant differences for PD $1.6-6.2(n=4, p=0.184)$.
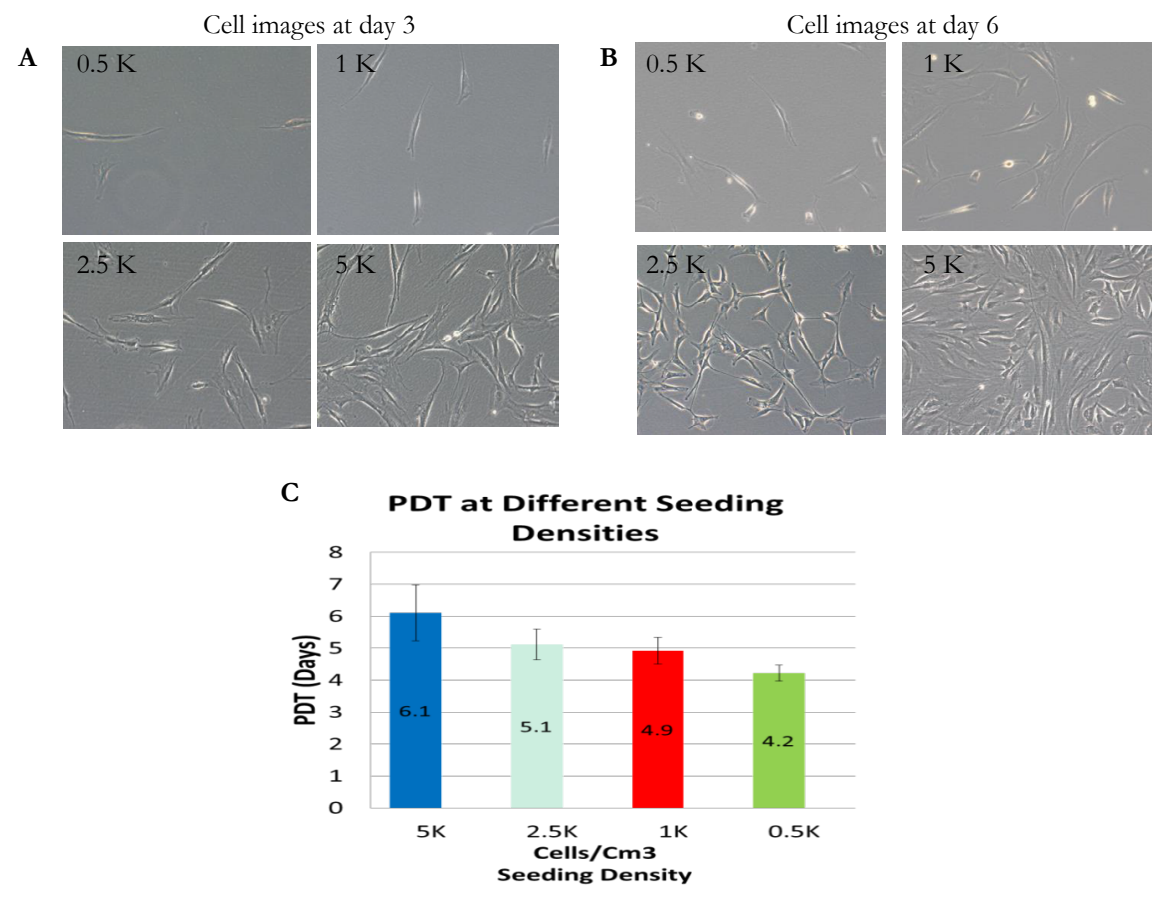

(Figure 2, panel C).

\section{The short term effect of PD on ASCs cell differentiation ca- pability}

Three ASCs were subjected to differentiation media towards to adipose, osteocyte, or cartilage at different PD stages as seeding density influenced the PD levels when the respective cultures reached $80 \%$ confluence for analyses. Adipogenic differentiation was evaluated by Oil red-O staining, a specific dye for lipid droplet (Figure 3, panel A). The Oil red-O staining intensities of adipogenic ASCs/undifferentiated ASCs were: 2.3 for PD 1.6; 2.8 for PD 3.8; 3.9 for PD4.9; and 1.7 for PD6.2 (Figure 3, panel B). There was no statistical significance for ASCs adipogenic differentiation rates at PD range from 1.6 to $6.2(n=3, p=0.256)$.

ASCs differentiation for osteogenic and chondrogenic phenotypes were evaluated by Alizarin Red staining and Alician blue staining, respectively (Figure 4 and 5). Representative images for osteogenic or chondrogenic differentiations at different PD groups were presented in panel A and quantified data for differentiation capabilities in panel B. There were no statistically significant differences in either Alizarin Red staining or Alician blue staining across tested PD ranges. The Alizarin Red staining intensities of osteogenic ASCs/undifferentiated ASCs were: 3.0 for PD1.6; 2.2 for PD3.8; 2.8 for PD4.9; and 3.7 for PD6.2. There was no statistical significance for ASCs osteogenic differentiation in the PD range from 1.6 to $6.2(n=3, p=0.472)$. The Alician blue staining intensities of chondrogenic ASCs/undifferentiated ASCs were: 1.9 for PD 1.6; 1.8 for PD 3.8; 2.1 for PD4.9; and 2.1 for PD6.2. There was no statistical significance for ASCs chondrogenic differentiation at PD range from 1.6 to $6.2(n=3, p=0.878)$.

In summary, cell growth and stem cell differentiation capability results demonstrated no significant changes of cell growth and differentiation rates for ASCs at PD range 1.6 to 6.2.

\section{Discussion}

Stems cells role in tissues regeneration, specifically ASCs role, has gained significant interest. ASCs present a particular advantageous stem cell population given ease of harvest and increased yield compared to other stem cell populations. In the United States there is a large interest in the potential clinical applications of ASCs, with countless articles published involving animal and in vitro experimentation. Though bone marrow derived stem cells have demonstrated intact DC following progressive in vitro expansion [23], ASCs have been shown to be more sensitive in vitro as demonstrated in previous experiments $[1,14-16,19]$. A standard and practical guideline for in vitro ASCs expansion is required to standardize results among worldwide laboratories.

Bieback et al., examined the effects of ASCs replicative aging using PD in the presence of human serum and fetal bovine serum on ASCs proliferation and adipogenic and osteogenic differentiation rates [20]. They reported that the PD of 6 and above was associated with decreased adipogenic differentiation rates using PD 6.0 as baseline in both growth media. Using flow cytometric markers, there were no differences in cell surface makers for immunophenotypes at PD 6.0 compare PD 0. Mechanistic evidence demonstrated increased expression of senescence markers werealso associated with PD beyond 6.0. They concluded that human serum was comparable to fetal bovine serum in long-term ASCs growth cultures.

Our interest was to initially reproduce their finding of diminished adipogenic differentiation rates associated with increased PD. In our one cell line subjected to long-term culture at $\mathrm{PD}>6.2$, phenotypic changes associated with increased senescence were 
Figure 3. Adipogenic differentiation capability of Adipose-derived stem cells (ASCs) at different population doubling number. Panel A: representative images of Oil Red-O staining; Panel B: bar graph of analyzed Oil Red-O staining data from three cell lines, adipogenic differentiation capability was measured by the intensity of Oil Red-O staining of adipogenic differentiated ASCs (cultured by adipogenic medium) over un-differentiated ASCs (cultured by m199 medium).There were no significantdifferences demonstrated for ASCs adipogenic differentiation rates at PD range from 1.6 to 6.2 ( $\mathrm{n}=3$, $\mathrm{p}=0.256$ ).
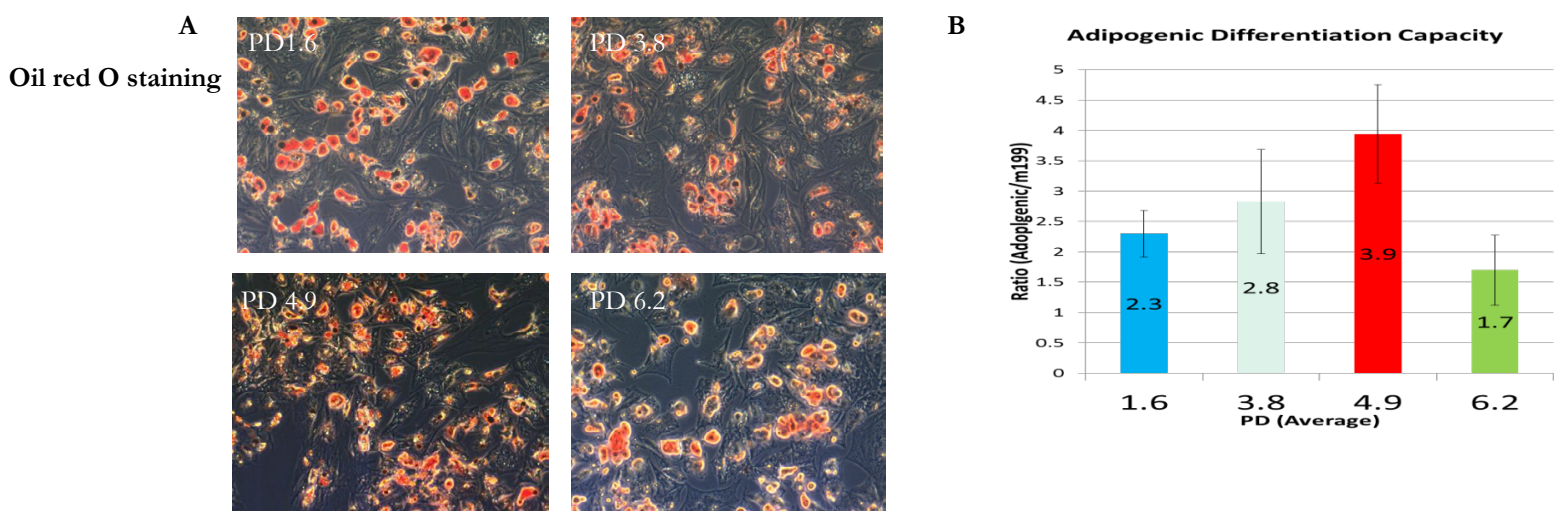

Figure 4. Osteogenic differentiation capability of Adipose-derived stem cells (ASCs) at different population doubling number. Panel A: representative images of Alizarin Red staining; Panel B: bar graph of analyzed Alizarin Red staining data from three cell lines, osteogenic differentiation capability was measured by the intensity of Alizarin Red staining of osteogenic differentiated ASCs (cultured by osteogenic medium) over un-differentiated ASCs (cultured by m199 medium).There were no significant differences demonstrated for ASCs osteogenic differentiation in the PD range from 1.6 to 6.2 ( $\mathrm{n}=3$, $\mathrm{p}=0.472)$.
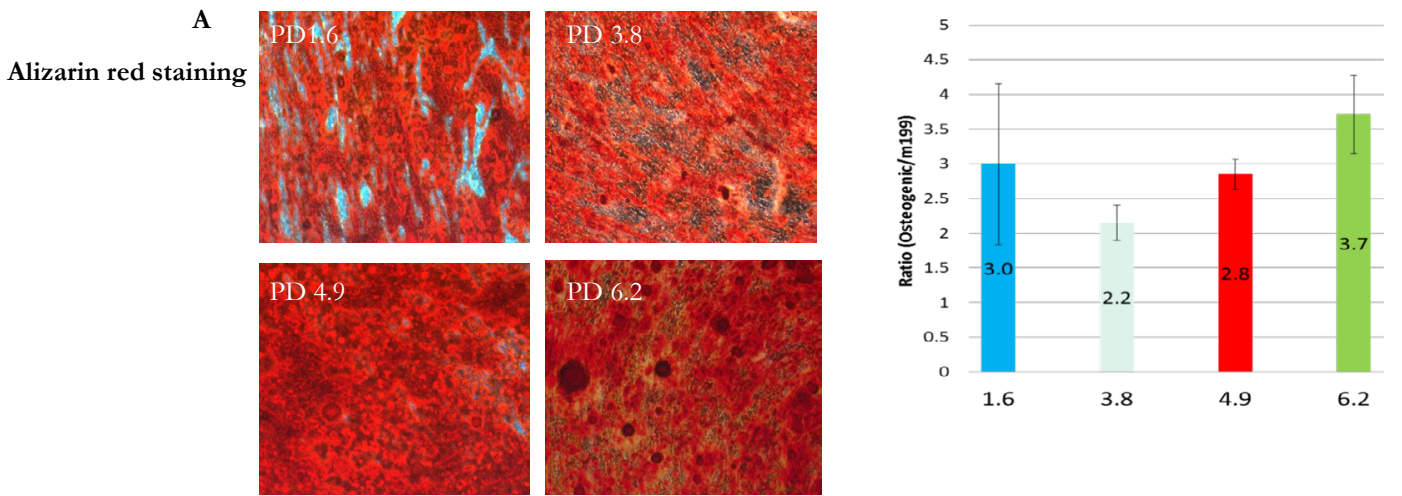

Figure 5. Chondrogenic differentiation capability of Adipose-derived stem cells (ASCs) at different population doubling number. Panel A: representative images of Alician blue staining; Panel B: bar graph of analyzed Alician blue staining data from three cell lines, chondrogenic differentiation capability was measured by the intensity of Alician blue staining of adipogenic differentiated ASCs (cultured by chondrogenic medium) over un-differentiated ASCs (cultured by m199 medium). There were no significant differences demonstrated for ASCs chondrogenic differentiation at PD range from 1.6 to 6.2 ( $\mathrm{n}=3$,

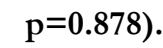
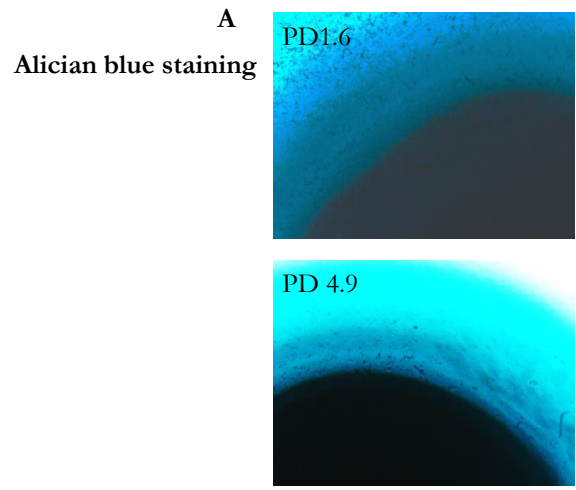
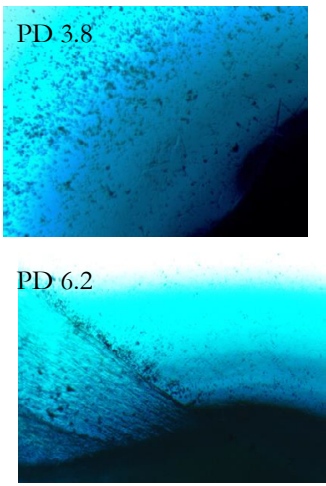

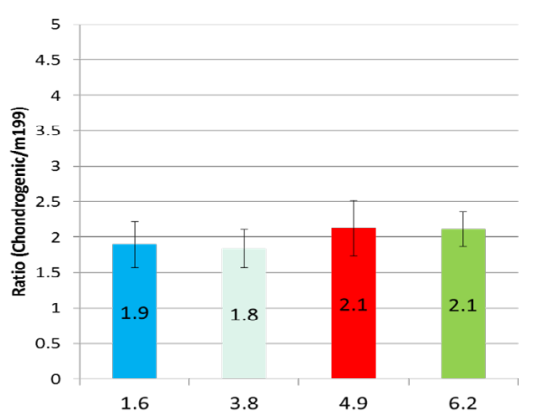

observed with significantly increased PDT. Our focus was then to understand the effects of replicative aging in PD from 0-6 as FDA approved clinical application would most likely require that cultured and expanded ASCs would not have decreased adipogenic differentiation capacity. The goal would be to generate an expanded ASCs culture specimen that would have similar properties to a minimally manipulated autologous FDA approved ASCs preparation.

Our short-term PD results demonstrated that ASCs populations 
may be expanded to a population doubling of 6.2 without any significant effect(s) on phenotypic growth rate or DC; beyond this PD level, increased PDT and decreased DC were demonstrated. Previous studies have demonstrated similar results which showed a trend towards increased passage numbers and decreased DC with increased in vitro culture [1, 14-16, 19, 20]. Comparing many previous studies which reported cell results as passage numbers compared to PD as in this study is difficult as the time to passage for most studies reported varies from 6 to 7 days with most PDTs for human ASCs reported in the 4 to 6 day range for passages less than 10. Comparing passage number to PD may be extrapolated by assuming that a given ASCs cell line test undergoes approximately 1.3 PDs for each passage it is subjected to, and thus passage 10 for reported cell population corresponds to a PD of approximately 13 . This is also variable due to tissue culture media conditions.

In examining canine ASCs, Lee et al., [3] demonstrated significantly increased PDT at PD greater than 6, with a trend towards increased PDT at lower PDs as well. In this study, there was also a dramatic decrease in DC with increasing PD. Di Battista et al., [1] demonstrated passage dependent osteogenic DC with human ASCs with almost complete phenotypic loss of osteogenic differentiation beyond PD 2. Interestingly, Di Battista also demonstrated some variance in PDT up to passage 6 (PD not reported) but no significant change similar to results demonstrated with our experiments.

Wang et al., [19] found that ASCs showed no significant difference in DC along adipogenic and osteogenic lineages or in common surface markers up to passage 15 (PD not reported), but did find that cells have significantly decreased immunomodulatory effect in both an in vivo murine peritonitis model and in immunomodulatory cytokine secretion at passage 3 compared to Passage 15. Their result lies in contrast to our results which showed decreased osteogenic DC beyond PD 6.1.

Safawani et al., [16] demonstrated preserved PDT at PD up to 15, but showed increased senesce markers and decreased DC along adipogenic and osteogenic lineages beyond PD 10. They concluded that ASCs likely present decreased clinical utility beyond this PD 10.

ASCs growth rate was reported to remain constant in early PD and slower with increased cell passaging $[14,15]$. A trend towards decreased DC along osteogenic and adipogenic DC was also demonstrated universally.

As ASCs were cultured over long-time intervals, in addition to changes in PDT and PD discussed, phenotypic appearance changes were noted as well. Across all cells lines with PD of 4 or greater, there was a noted transition to a more fibroblast like, spindle shaped phenotype. This characteristic has been observed in previous studies examining long-term in vitro cultures of ASCs, but the trend was even more pronounced in the cells initially seeded at lower seeding density $\left(500\right.$ cells $/ \mathrm{cm}^{2}$ and 1000 cells $/ \mathrm{cm}^{2}$ ) compared to higher seeding density $\left(2500 \mathrm{cells} / \mathrm{cm}^{2}\right.$ and 5000 cells $/ \mathrm{cm}^{2}$ ). There were no changes in DC or PDT for PD less than 6.2, but the fibroblast like phenotype was more evident at PD greater than 6.2.
Almost universally in bench, animal, and clinical experiments in which ASCs have been utilized, in vitro expansion is necessary to obtain a sufficient cell population prior to use $[3,13]$. Future studies are needed to examine if the type of serum utilized effects ASCs phenotypical or genotypic expression. Also, future studies should examine and report in PD as opposed to nonspecific passage, to allow direct comparison of results across studies. Another potential area for exploration involves the estimation of ASCs yield for clinical application (see Table 1).

In a summary, no significant differences in growth rates or DC were observed among individual specimens and overall for in vitro expansion of ASCs up to PD 6.2. A trend towards decreased growth rates and DC were demonstratedwith greater than PD 6.2. Our data suggest that future ASCs clinical applications that require in vitro expansion may consider expanded ASCs up to but not exceed PD 6.2. Standardization of in vitro expanded ASCs usage in clinical translational applications requires validation and use of specific PD instead of the non-specific passage numbers which are currently widely reported.

\section{Acknowledgement and Declarations}

Special thanks for the support by the Surgery Department at Cooper University Hospital and our research manager, Denise Schmidt. The authors listed above certify that they have NO affiliations with or involvement in any organization or entity with any financial interest (such as honoraria; educational grants; participation in speakers' bureaus; membership, employment, consultancies, stock ownership, or other equity interest; and expert testimony or patent-licensing arrangements), or non-financial interest (such as personal or professional relationships, affiliations, knowledge or beliefs) in the subject matter or materials discussed in this manuscript.

\section{References}

[1]. Di Battista JA, Shebaby W, Kizilay O, Hamade E, Abou Merhi R, et al. (2014) Proliferation and differentiation of human adipose-derived mesenchymal stem cells (ASCs) into osteoblastic lineage are passage dependent. Inflamm Res 63 (11): 907-917.

[2]. Turner NJ, Badylak SF (2012) Regeneration of skeletal muscle. Cell Tissue Res 347(3): 759-774.

[3]. Lee KS, Kang HW, Lee HT, Kim HJ, Kim CL, et al., (2014) Sequential sub-passage decreases the differentiation potential of canine adipose-derived mesenchymal stem cells. Res Vet Sci 96(2): 267-275.

[4]. Tsuji W, Rubin JP, Marra KG (2014) Adipose-derived stem cells: Implications in tissue regeneration. World J Stem Cells 6(3): 312-321.

[5]. Gir P, Oni G, Brown SA, Mojallal A, Rohrich RJ (2012) Human adipose stem cells: current clinical applications. Plast Reconstr Surg 129(6): 1277 1290 .

[6]. Marx C, Silveira MD, Beyer Nardi N (2015) Adipose-derived stem cells in veterinary medicine: characterization and therapeutic applications. Stem Cells Dev 24(7): 803-813.

[7]. Lensch MW, Daheron L, Schlaeger TM (2006) Pluripotent stem cells and their niches. Stem Cell Rev 2(3): 185-201.

[8]. Ross RJ, Shayan R, Mutimer KL, Ashton MW (2014) Autologous fat grafting: current state of the art and critical review. Ann Plast Surg 73(3): 352357.

[9]. Lee EM, Kim AY, Lee EJ, Park JK, Lee MM, etal. (2015) Therapeutic Effects of Mouse Adipose-derived Stem Cells and Losartan in the Skeletal Muscle of Injured Mdx mice. Cell Transplant 24(5): 939-953.

[10]. Pilia M, McDaniel JS, Guda T, Chen XK, Rhoads RP, et al., (2014) Transplantation and perfusion of microvascular fragments in a rodent model of volumetric muscle loss injury. Eur Cell Mater 28: 11-24.

[11]. Abdeen AA, Weiss JB, Lee J, Kilian KA (2014) Matrix Composition and Mechanics Direct Proangiogenic Signaling from Mesenchymal Stem Cells. 
Tissue Eng Part A 20 (19-20): 2737-2745.

[12]. Lindroos B, Suuronen R, Miettinen S (2011) The potential of adipose stem cells in regenerative medicine. Stem Cell Rev 7(2) 269-291.

[13]. Zhao Y, Waldman SD, Flynn LE (2012) The effect of serial passaging on the proliferation and differentiation of bovine adipose-derived stem cells. Cells Tissues Organs 195(5): 414-427.

[14]. Gruber HE, Somayaji S, Riley F, Hoelscher GL, Norton HJ, et al., (2012) Human adipose-derived mesenchymal stem cells: serial passaging, doubling time and cell senescence. Biotech Histochem 87(4): 303-311.

[15]. Wall ME, Bernacki SH, Loboa EG (2007) Effects of serial passaging on the adipogenic and osteogenic differentiation potential of adipose-derived human mesenchymal stem cells. Tissue Eng 13(6): 1291-1298.

[16]. Safwani WK, Makpol S, Sathapan S, Chua KH (2012) The impact of longterm in vitro expansion on the senescence-associated markers of human adipose-derived stem cells. Appl Biochem Biotechnol 166(8): 2101-2113.

[17]. Balducci L, Blasi A, Saldarelli M, Soleti A, Pessina A, et al., (2014) Immortalization of human adipose-derived stromal cells: production of cell lines with high growth rate, mesenchymal marker expression and capability to secrete high levels of angiogenic factors. Stem Cell Res Ther 5(3): 63.

[18]. Koellensperger E, Bollinger N, Dexheimer V, Gramley F, Germann G, et al., (2014) Choosing the right type of serum for different applications of human adipose tissue-derived stem cells: influence on proliferation and differentiation abilities. Cytotherapy 16(6): 789-799.

[19]. Wang X, Liu C, Li S, Xu Y, Chen P, et al., (2015) Effects of continuous pas- sage on immunomodulatory properties of human adipose-derived stem cells. Cell Tissue Bank 16(1): 143-150.

[20]. Bieback K, Hecker A, Schlecter T, Hofman I, Brousos N, et al., (2012) Replicative aging and differentiation potential of human adipose tissue-derived mesenchymal stronmal cells expanded in pooled human or fetal bovine serum. Cytotherapy14(5): 570-583.

[21]. Minteer DM, Marra KG, Rubin JP (2015) Adipose Stem Cells: Biology, Safety, Regulation, and Regenerative Potential. Clin Plast Surg 42(2): 169179.

[22]. Naghshineh N, Brown S, Cederna PS, Levi B, Lisiecki J, et al., (2014) Demystifying the U.S. Food and Drug Administration: understanding regulatory pathways. Plast Reconstr Surg 134(3): 559-569.

[23]. Jaiswal N, Haynesworth SE, Caplan AI, Bruder SP (1997) Osteogenic differentiation of purified, culture-expanded human mesenchymal stem cells in vitro. J Cell Biochem 64(2): 295-312.

Special Issue on
"Mesenchymal Stem Cells"
Edited by:
Marcio Alvarez-Silva, Universidade Federal de Santa
Catarina, Brazil.
E-mail: marcio.alvarez@ufsc.br

